\title{
Small Tumors as Risk Factors not Disease
}

\author{
Peter H. Schwartz \\ Indiana University Center for Bioethics \\ Indiana University School of Medicine \\ Indiana University - Purdue University, Indianapolis
}

2014

[Preprint]

\section{Philosophy of Science}

\section{Address:}

IU Center for Bioethics

410 W. $10^{\text {th }}$ St., Ste. 3100

Indianapolis, IN 46202

\begin{abstract}
:
I argue that ductal carcinoma in situ (DCIS), the tumor most commonly diagnosed by breast mammography, cannot be confidently classified as cancer, i.e. as pathological. This is because there may not be dysfunction present in DCIS - as I argue based on its high prevalence and the small amount of risk it conveys - and thus DCIS may not count as a disease by dysfunction-requiring approaches, such as Boorse's Biostatistical Theory and Wakefield's Harmful Dysfunction Account. Patients should decide about treatment for DCIS based on the risks it poses and the risks and benefits of treatment, not on its disease status.
\end{abstract}

\section{Acknowledgments:}

Thanks to Chris Boorse, Dan Hausman, Eric Meslin, and Greg Sachs for discussion. Thanks to Anya Plutynski for inviting me to participate in this panel and Miriam Solomon for her commentary. My research was supported in part by a career development award from the American Cancer Society (CCCDA-10-085-01) and by the Indiana Clinical and Translational Sciences Institute (NIH/ NCRR: 1 UL TR001108-01). 


\section{Small Tumors as Risk Factors not Disease}

\section{Introduction:}

In this paper I argue that it is unclear whether ductal carcinoma in situ (DCIS), the tumor most commonly diagnosed by breast mammography, is really a cancer, i.e. pathological. In particular, it is not clear that there is dysfunction present in DCIS, and thus it is not clear if it counts as a disease according to a dysfunction-requiring account such as Christopher Boorse's Biostatistical Theory (Boorse 1977, 1987, 1997) or Jerome Wakefield's Harmful Dysfunction Account (Wakefield 1992a, b). This uncertainty stems from the high prevalence of DCIS and the limited amount of risk that it conveys.

In the end, I will argue, we may infer the presence of a system that is functioning below average but perhaps not poorly enough to count as dysfunctioning. The question of how to classify DCIS reflects the "line drawing problem" for determining when dysfunction is present (Schwartz 2007b, Hausman 2012). At the same time, the question of whether doctors should offer treatment to patients with DCIS does not depend on whether DCIS is a disease, but instead on whether the benefits of treatment outweigh the burdens or risks (Vickers, Basch, and Kattan 2008)(Omer et al. 2013).

\section{Risk-Based Diseases and the Biostatistical Theory (BST)}

I have argued that most people diagnosed with high blood pressure (hypertension) and high cholesterol (hypercholesterolemia) are actually healthy, i.e. have no pathological condition, but instead have a risk factor for future disease, such as stroke, heart attack, and other conditions (Schwartz 2008). Given a “dysfunction-requiring” theory such as the Biostatistical Theory (Boorse 1977, 1987, 1997) or the Harm Dysfunction Account (Wakefield 1992a, b) a condition can only count as a disease if there is biological dysfunction present. And I have argued that for hypertension and hypercholesterolemia there is no reason to conclude that a part is dysfunctioning, i.e. failing to carry out its function (Schwartz 2008).

While my previous discussion focused on high blood pressure and high cholesterol, it appears that similar points can be made for other "risk-based" diseases, including diabetes, osteoporosis, and obesity (Schwartz 2008). In all these cases, the process by which medical science determines the existence and boundaries of the disease and indication for treatment is very clear. Epidemiological or observational research shows a correlation between some parameter (blood pressure, serum cholesterol, blood sugar, bone mineralization, or body mass index) and the chance of developing a certain disease in the future. Then a clinical study, hopefully at least one randomized, controlled, double-blinded trial, shows that among individuals whose parameter falls above a certain line (e.g. a systolic blood pressure above $160 \mathrm{~mm} \mathrm{Hg}$ ), those that get a certain treatment have a lower risk of a future disease than those who get a placebo (Greene 2007, Chobanian et al. 2003, Westin and Heath 2005).

Of the two most prominent dysfunction-requiring theories, I will focus here, as previously, on applying Boorse's Biostatistical Theory, whose three key definitions are as follows:

1. The reference class is a natural class of organisms of uniform functional design; specifically, an age group of a sex of a species. 
2. A normal function of a part or process within members of the reference class is a statistically typical contribution by it to their individual survival and reproduction.

3. A disease is a type of internal state which is either

[a] an impairment of normal functional ability, i.e. a reduction of one or more functional abilities below typical efficiency, or

[b] a limitation on functional ability caused by environmental agents.

(1997, 7-8, formatting and numbering added under (3))

Most conditions are classified as disease based on criterion (3a). Take congestive heart failure (CHF) in a 50-year-old man, for instance. The human heart counts as having the function of pumping blood since this is what it does that typically contributes to survival and reproduction in 50-year-old men (Premises 1 and 2). Assume that the heart in a typical 50-year-old man has an "ejection fraction" (EF) the percent of blood pushed out by each contraction - of $50 \%$, and that the heart of the individual with CHF has an EF of just 15\%. Thus his heart is beating significantly "below typical efficiency" and counts as pathological according to Premise (3a).

As I argued previously (Schwartz 2008), when one looks at risk-based diseases like hypertension, it seems that the relevant organs are carrying out their typical functions with adequate effectiveness. For example, the combination of organs and feedback systems that determine blood pressure - including the cardiovascular, excretory, and endocrine systems - has at least one very clear function: keeping the pressure high enough that organs remain perfused, without becoming so high that the pressure interferes with perfusion or causes immediate damage. And the blood pressure in individuals with Stage 1 hypertension is in a range that allows all these things to happen (Systolic blood pressure 140-160 mm Hg; Diastolic blood pressure 80-90 mm Hg).

The only possibility of inferring the presence of dysfunction in the case of Stage 1 hypertension would be in relation to another function that one might assign to the blood pressure system, i.e. of keeping blood pressure in a range that will minimize the chance of future heart attack or stroke. While I have argued that this is a somewhat peculiar sounding function (Schwartz 2008), let's assume for now that it does qualify as a function by Premise 2 of the BST (c.f. Boorse 2012, manuscript). Then the question is whether the organs and feedback systems that determine blood pressure should be counted as failing to carry out this function adequately in patients with Stage 1 hypertension.

But answering this question raises complex questions (Schwartz 2007b). Here's the issue: For some token part or process to count as dysfunctioning, it is not enough if it is just carrying out some function below an average level for individuals of that age and gender. Go back to the example of heart beating and CHF: Some 50 year old men with healthy hearts may have an EF as high as $60 \%$ or as low as $40 \%$, based on genetics or training, etc. And while an EF of $40 \%$ may fall below average for this reference class, and may carry some undesirable consequences (such as inability to achieve certain feats of endurance), it may still count as falling within the range of normal variation, and not as dysfunctional. Clearly, functioning must fall significantly below the typical level to count as dysfunctional; otherwise, all individuals below average would count as diseased. 
Somewhere in the continuum we must draw a line where "normal variation" ends and where dysfunction begins (Schwartz 2007b).

The two key factors in drawing this line appear to be some combination of prevalence and severity of negative impact. In his comments on this topic, Boorse suggests that only prevalence matters (c.f., 1987, 370; 1997, 8). According to this approach, determining whether a level of functioning counts as dysfunctional just as long as it is below average and expressed by a very few members of the reference class. The attraction of the prevalence view is that it doesn't seem to require any judgment about the value of the level of functioning, which fits with Boorse's naturalistic approach. Boorse admits that the location of the precise line is "arbitrary" $(1987,371)$ and "can only be conventionally chosen” $(1977,559)$.

Even with these caveats, a prevalence approach cannot work, I have argued (Schwartz 2007b). For at least some traits and some reference classes, even a low level of functioning that is extremely rare may not be pathological. For instance, if among 20year-old men, the highest functional efficiency is $65 \%$ and the lowest is $58 \%$, and all can carry out all standard activities with no problem, there is no reason to assume that any of them have congestive heart failure (the "problem of healthy populations") (Schwartz 2007b).

At the same time, for other traits and reference classes, many more than just the lowest functioning few in the reference class may count as dysfunctioning (the "problem of common diseases"). For example, the prevalence of serious dysfunction of the hip joint, termed "canine hip dysplasia" is $30 \%$ in some breeds of dogs; Alzheimer's disease occurs in 16\% in people over 85 years old (see Schwartz 2007b). Boorse did allow that some levels of functioning could count as dysfunction not based on their prevalence but instead because they are caused by "environmental agents" (1997, 8; Premise 3b, above). But these two conditions do not seem to be clearly of that sort.

It appears that, in addition to considering prevalence, there must be some judgment about whether the level of functioning of the trait token has significant negative consequences (Schwartz 2007b). If a level of functioning has serious negative consequences, then even if it is relatively common, it can count as dysfunctioning. If a level of functioning has no negative consequences, then it should not count as dysfunction, even if this is the lowest level in a given reference class, expressed by just 1 in 1,000,000 individuals in that reference class. There are some problems with this approach, and Dan Hausman has recently made excellent suggestions regarding this (Hausman 2012).

Without delving more deeply into these issues, we can conclude that determining whether a blood pressure should be counted as reflecting dysfunction or not must depend on some mix of prevalence and negative consequences. And by this test, it appears that in many cases of Stage 1 hypertension, there will be no good reason to infer the presence of dysfunction. For example, a 60-year-old man with blood pressure 145/70 counts as having Stage 1 hypertension by current guidelines and should be started on treatment. But this blood pressure is at the median for men his age and conveys a minimal increase in risk over what it would be if his blood pressure were 120/50, which is optimal (Chobanian et al. 2003).

What matters to the authors of guidelines is the very simple fact that a blood pressure of this level puts him in a group where we know with relative certainty that he 
can safely reduce his chance of a cardiovascular event if he takes certain pills. The comparison blood pressure and risk is the lower blood pressure he will have on these pills, not anything about the blood pressure of other members of this reference class.

Similar arguments apply to the condition of high cholesterol. Based on research showing beneficial effects of starting a "statin" medication, a 60-year-old man with a low density lipoprotein (LDL) of $105 \mathrm{mg} / \mathrm{dL}$ and certain other risk factors may count as having "high cholesterol." But at the same time, this LDL is significantly below the median for this reference group and is probably not even that much higher than in primitive populations (National Cholesterol Education Program (NCEP) Expert Panel on Detection 2002)(Westin and Heath 2005).

Accounts that do not require the presence of dysfunction for disease may conclude that both hypertension and hypercholesterolemia are pathological. For instance, according to the malady account (Clouser, Culver, and Gert 1981), high cholesterol or high blood pressure may well count as pathology due the increased risk they convey. That said, such accounts are generally more liberal in identifying the presence of disease, and thus are subject to counterexamples such as classifying pregnancy as disease (Boorse 1997, Schwartz 2007a).

Note as well that giving up calling these conditions diseases has no impact on the moral imperative for doctors to diagnose and treat them. Most accounts of the goals of medicine recognizes that contributing to wellbeing by preventing a case of disease is just as important and admirable as contributing to wellbeing by treating or ameliorating the effects of a disease (Daniels 2008, Miller and Brody 2001). In short, there is nothing wrong with offering people a pill to reduce their risk of cardiovascular disease, but this should not be equated with diagnosing them with a disease.

\section{Screening and Asymptomatic Cancers}

A natural next question is whether asymptomatic cancers detected by cancer screening tests are diseases or not. All screening tests - including mammograms for breast cancer, colonoscopies for colon cancer, prostate specific antigen for prostate cancer, and pap smears for cervical cancer - identify many tumors that are too small to feel and are not causing any problem. In each case, the presence of the small tumor is not interfering with the functioning of the organ involved. For instance, for a 60-year-old man, a small prostate cancer does not interfere with the prostate gland making its usual contributions to his survival and reproduction; the man's ability to father a child is not affected. The breast of a 50-year-old woman typically makes no contribution to survival and reproduction, due to the unlikelihood of nursing a child at this age, and, anyway, a breast with a small breast cancer can produce milk just as well as one without a small cancer. Only when a cancer spreads or metastasizes does it cause clear dysfunction.

Finally, in a key analogy, screening tests are justified on the same basis as treatments for risk-based diseases like hypertension and high cholesterol: tests are recommended based on evidence from randomized, controlled trials that undergoing screening and follow up care (including removing asymptomatic tumors) reduces mortality and morbidity (USPSTF 2008).

If one is going to make a case for the presence of dysfunction in the case of asymptomatic tumors, one must look at the functioning of cellular processes that contribute to survival and reproduction by blocking unrestrained growth and spread of 
cells (i.e. cancer). The natural history of cancer is well understood: it begins with a single cell that undergoes some changes - either in a gene or genes, or involving some protein or gene product - that leads it to start dividing. As the cell's progeny continue to divide, some acquire additional changes that confer the ability to divide more actively and to spread in that organ and metastasize to other organs, where it eventually maims or kills. A tumor will often become palpable only once it has grown to $10^{9}$ cells, and at this size often some cells have already spread elsewhere, so removing the primary tumor no longer produces cure. Screening technologies try to find tumors when they are smaller, sometimes $10^{6}$ cells, either before they become cancerous or before they have spread, when treatment can be easier and more effective (Croswell, Brawley, and Kramer 2011).

With this picture in mind, we can see why one might infer that even small tumors should be classified as pathological according to a dysfunction-requiring account. The changes that lead the cell to change in shape, start dividing, and acquire the ability to spread may be assumed to involve the dysfunctioning of systems that typically block the changes that turn the cell into a killing machine.

To examine this picture in some more detail, consider the following: All cells in the body arise from growth and differentiation from the single cell of the fertilized egg. In each tissue, specific changes occur that lead the cells to differentiate and usually stop dividing. And the resulting aspects of genetics or cellular machinery can be seen as contributing to survival and reproduction by causing the differentiation and restraining further division, and thus have this function as their function, by Boorse's Premise 2.

In real life, this is all incredibly complex and varies from tissue to tissue, or course. But assume for simplicity that differentiation and non-division in some tissue is brought about by turning off two genes (call them $a$ and $b$ ) and turning on two others (call them $c$ and $d$ ). Thus, we might characterize the Differentiation and non-Division (DD) system in this cell as involving the following all four of these genes, as well as any other genes and proteins that they interact with to cause differentiation and to restrain division. On the simplest conception, then, when a cell of this type starts to divide and dedifferentiate, some element of the DD system may be assumed to be malfunctioning. For instance, perhaps $a$ and $b$ start being transcribed and translated into proteins again, due to failure of the mechanisms that usually keeps them from doing so. Or $c$ or $d$ stop making their proteins, or other changes in the cell block their proteins from having their usual effects.

One may resist this inference that dysfunction is occurring by raising the possibility that the dedifferentiation and division may be occurring due to cellular changes that have nothing to do with the elements of DD listed above. Maybe, for instance, $a$ has not started making its gene product, but some other gene, call it $e$, which is usually not involved, has mutated (call it $e^{\prime}$ ) and is now producing a novel protein that has the same effect as $a$ 's gene product. In other words, DD is functioning normally, but dedifferentiation and division are occurring due to the action of something outside that system.

At the same time, there appear to be two ways to defend the inference of dysfunction, even in such cases. First, the ability of $e$ ' to stimulate dedifferentiation and division may be seen as interfering with a broader system that involves DD but also other elements of the cell (call it DD+). DD+ would certainly also count as having the function 
of maintaining differentiation and restraining division, and this broader system may be seen as dysfunctioning, due to e', even if DD is not.

Second, even without defining a broader system DD+, one might conclude, given

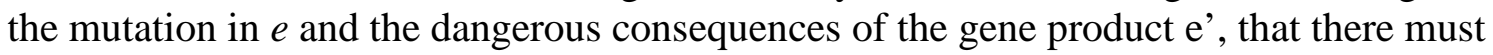
be at least some signaling system or other cellular process that is failing to carry out its usual function, i.e. make its usual contribution to survival and reproduction.

At this point, we've gotten a bit removed from specific biological examples, so I'm going to leave that discussion there. For the rest of this paper, I'll assume that we can assume that there is a system that is functioning below its optimal level, at least, when dedifferentiation and division of the cell occurs.

Then we can go back to the question that started this section: When does the decreased functioning of DD count as dysfunction, rather than just below-average or suboptimal functioning? Is it at the point when gene $a$ or $b$ turn on, even a little bit? Or when $c$ or $d$ turn off, or just don't produce at the highest level, or their gene products have somewhat diminished efficacy at maintaining differentiation and non-division?

To put it in terms of the changes in the cell: Can we conclude that DD is dysfunctioning if the cell has become just slightly less differentiated than other cells of the same type? Once it starts dividing? Once it is dividing and has also acquired additional changes? Or only after it is dividing and has acquired the ability to invade tissue and spread to other organs, the signs of truly dangerous cancer?

I will argue that it is quite hard to draw a line in this progression where suboptimal or below-average functioning of the DD system has transitioned over to dysfunctioning. One could draw the line at the first change, perhaps when dividing has started to occur. Or one could make an argument, in at least some cases, for holding off until the final step, once the cell has acquired the ability to invade tissue and metastasize. I will argue that many of these ways of drawing the line are acceptable and make no appreciable difference in the practice of medicine. It makes the difference between whether intervention counts as treatment or prevention, but not whether such interventions should be covered be carried out by physicians or covered by health insurance.

At the same time, biological science and pathology depend on drawing this line, since it determines what counts as normal or healthy and what abnormal and unhealthy. And despite the fact that the location of the line is somewhat arbitrary, I will argue that it is a necessary part of biological science to choose one of the spots.

\section{Ductal Carcinoma in Situ (DCIS):}

To consider this question more closely, I'll focus on the example of ductal carcinoma in situ (DCIS), a type of breast cancer that has taken on great importance with the advent of widespread mammographic screening. DCIS has "all the morphologic features of malignancy, but without any evidence of basement membrane penetration" (Erbas et al. 2006, 136). It falls on a continuum from normal cells in the epithelium lining the ducts, to atypical ductal hyperplasia made up of cells that are dividing and starting to look abnormal, to DCIS when they are starting to look just like cancer cells but have not penetrated the basement membrane. When the cells penetrate the basement membrane, they become invasive ductal carcinoma (Erbas et al. 2006). 
This is a cancer that was diagnosed relatively rarely in the era before mammography - in 1973, the incidence rate for women was 2.4 per 100,000 per year but mammography made more common: in 1992 it had risen to 15.8 per 100,000 (Erbas et al. 2006, Ernster et al. 1996). Out of all the tumors identified in mammography screening programs, DCIS makes up about $15-25 \%$ of them. And when DCIS is found, it is treated by lumpectomy or mastectomy, sometimes with chemotherapy or radiation, and these treatments have been shown to diminish the risk of developing IDC in that breast (Kuerer et al. 2009).

The progression from normal epithelial cells, to ADH, to DCIS, and then to IDC thus provides a real-life example of the sort of sequence we discussed in the model of the last section. And for each step in this sequence we may ask whether that type of cell should be classified as pathology, i.e. a case where dysfunction can be inferred, or as a normal type of cell, admittedly one that has some increased chance of progressing to cancer in a certain time frame. Even at the first step, when ADH develops, one might infer that the DD system is no longer functioning as well as it did when they were normal. But has it crossed over from suboptimal functioning, perhaps below-average functioning, to dysfunctioning? We can ask this again as the cell becomes DCIS and then IDC.

As discussed above, answering this line-drawing problem appears to depend on both the prevalence and negative consequences of each condition (Schwartz 2007b). And for each condition, the negative consequences have to be understood in terms of the risk the lesion will progress, spread, and cause morbidity and mortality.

Let's talk about prevalence first. As mentioned above, the rate at which DCIS is diagnosed is quite low; even with a universal screening program, the rate is under 16 per 100,000 (Erbas et al. 2006). But the question of how much undiagnosed DCIS is out there has to come from pathological studies of breasts of women who die from other causes. These studies vary along a number of dimensions, including the age of the women, the type of autopsy (including the thickness of slicing), and the criteria used. One review of a range of studies points to a median prevalence of $8.9 \%$ (Welch and Black 1997). The data for the prevalence of ADH is also complicated and inconclusive (Erbas et al. 2006), but it is not unreasonable to assume it is about twice that of DCIS. For the rest of this paper, I'm going to focus on the reference class of women who are 50 years old and assume that the prevalence of DCIS is $10 \%$ and ADH is $20 \%$, with "normal cells" making up the other $70 \%$.

Now for risk. First, it's crucial to remember that even if untreated, DCIS does not always progress to breast cancer. A woman with DCIS may die for other reasons before it progresses, or the lesion may not progress, or the breast tissue involved may "involute," before the DCIS progresses, eliminating it (Erbas et al. 2006). But assuming a 50 year old woman has DCIS and that she lives 20 years, how often will the DCIS progress to IDC? Again, the science is inconclusive and variable. The best data comes from studies where researchers reviewed biopsy specimens that were read as "normal" and identify those where DCIS was missed, and then contact those women. One large review of almost 12,000 biopsies found 28 cases misread in this way, and that cancer had developed in 7 of them within 15 years (25\%) (Page et al. 1982). Another review of over 9000 biopsies found 80 that harbored DCIS, and of these 11 (13.7\%) had developed cancer over an average of 17.5 years (Eusebi et al. 1994). To simplify for the purpose of 
this paper, I'm going to assume that for a 50-year-old woman, in general, untreated DCIS has an average chance of about 25\% of progressing over 20 years to invasive cancer.

So, if a 50-year-old woman learns that she has an area of DCIS, how much of an increased risk does she have than the baseline case? At least one model suggests that a typical 50-year-old woman has about a 3.5\% chance of developing palpable or symptomatic DCIS by the age of 70. Let's use that number for our baseline.

The final challenge is to estimate the risk of ADH progressing to DCIS and then IDC. There's no simple way to estimate or interpolate this risk; for the purposes of discussion, let's assume it's about $10 \%$. This produces the following picture of prevalence and risk (Table 1):

Table 1. Estimates of prevalence and chance of progression

\section{Prevalence among 50-year-old women Chance of progression to sIDC in 20 years} $($ sIDC = symptomatic or palpable IDC)

$\begin{array}{lll} & \text { Cell Type } & \\ \text { Normal } & \text { ADH } & \text { DCIS } \\ 70 \% & 20 \% & 10 \% \\ 3.5 \% & 10 \% & 25 \%\end{array}$

As mentioned above, and with these numbers fleshing out the picture, we can see that if prevalence in the reference class fully determines the line between low normal and abnormal levels of functioning, then even DCIS will not harbor a dysfunctional DD system. Prevalence-focused approaches generally focus on levels of functioning that are in just the lowest few percentile for a reference class of individuals, i.e. about two standard deviations from the mean given a normal distribution (Boorse 1987, 370; 1997, 8). And DCIS is present (we are assuming) in about $10 \%$ of 50 -year-old women.

But now let's turn to the magnitude of negative consequences. In the case of DCIS, it is hard to determine whether the increase in risk is large enough to justify the conclusion that the DD system is dysfunctioning. On the one hand, a 25\% risk of progression to symptomatic IDC over 20 years is impressive; on the other hand, fully $75 \%$ of women with DCIS, according to this assumption, will not develop IDC in this time period. Do they have a dysfunction that has not led to negative consequences, or a system that is functioning at relatively low levels, not yet dysfunctional?

There are no clear guidelines for such judgment. Similarly for ADH, there may be significant vagueness about whether the DD system is dysfunctional. Compared to DCIS, prevalence is higher and the risk of progression to IDC is lower, but even here there has been a three-fold increase over the baseline case. The DD system is not functioning optimally, and it seems that one could make the case for seeing it as dysfunctional even at this point.

At the same time, one could go the other way and resist concluding that dysfunction is present until actual IDC has developed. As mentioned above, autopsy studies suggest that fully $1.3 \%$ of women have IDC. These are tumors that were undetected when the woman was alive, so we may assume that many of them are not yet 
sIDC, i.e. not yet palpable or symptomatic. If the woman had lived, presumably there would have been a reasonably high chance of progression and spread over some time period.

Remember that we are assuming, based on the best evidence available, that DCIS has a $25 \%$ of progression to symptomatic IDC. Of course, even without harboring any DCIS cells, a 50 year old woman may have this sort of risk of developing IDC in the next 20 years, based on factors such as family history, genetics, age of menarche, number of children etc. (Online calculators are available to calculate the risk, based on epidemiological data.) Presumably, if the risk that DCIS conveys justifies the conclusion that the DD system is dysfunctioning, the cellular or genetic characteristics that convey this risk may count as disease as well, even without any pathological change in the appearance of the cells.

\section{Conclusion:}

My main conclusion, then, is that there is significant uncertainty about whether DCIS can be inferred to harbor a dysfunctional DD system. Instead, it appears possible to assert or deny that dysfunction is present in ADH, DCIS, and perhaps even asymptomatic IDC. There is a line-drawing question here, about when the functioning of the DD system should be counted as dysfunctioning vs. simply suboptimal or below average.

Science now understands much more about the progression of normal cells to cancer and the prevalence and risk associated with each stage. Much more needs to be done to identify the specific molecular changes involved and the heterogeneity of each of the currently recognized cell types. Discoveries in this area will allow doctors to do better at predicting individuals' risk of developing cancer, and such information may inform decision making about possible preventive steps, whether or not the condition is itself pathological or not.

As mentioned above for the case of high blood pressure and high cholesterol, counting DCIS as a disease or not does not change whether most systems of healthcare ethics or justice would see its treatment as justified. Even if DCIS is not a disease, as long as treating it can significantly reduce the risk of developing symptomatic IDC, and can do so safely and with acceptable expense and burden to the patient, then that treatment is justified as prevention.

From this perspective, deciding whether DCIS counts as disease may be seen as distracting from the key questions involved in evaluating preventive care (Vickers, Basch, and Kattan 2008). And this is certainly right, to some extent: the judgment of whether a condition is a disease is separate from whether it carries risks and whether there are treatments that can reduce that risk without imposing unacceptable burdens, cost, or new risks. In fact, simply describing DCIS as a disease, if done without emphasizing the assessment of risk and benefit in choosing treatment, might interfere with helping individuals determine whether to undertake certain steps for risk reduction. A recent study has shown that describing DCIS as a disease has a significant impact on patient decisions about treatment, even if there is no change in how the risks and benefits of treatment are described (Omer et al. 2013).

At the same time, this conclusion does not support the view that defining disease has become entirely irrelevant to directing medical care (Vickers, Basch, and Kattan 
2008). Even if it doesn't matter if DCIS is a disease, it still matters that at least symptomatic IDC is a disease, and that the treatment of DCIS is a form of disease prevention. Medicine has long identified itself as focused on the prevention and treatment of disease, and good arguments have been offered for continuing this focus (Daniels 2008). Seeing medicine in this way relies on being able to draw lines between normal, healthy conditions and abnormal or pathological ones, and while there are complexities and challenges to such line drawing, it is still central, and essential, to medicine. 


\section{References:}

Boorse, Christopher. 1977. "Health as a Theoretical Concept." Philosophy of Science 44 (4):542-573.

1987. "Concepts of Health." In Health Care Ethics: An Introduction, edited by D. VanDeVeer and T. Regan, 359-393. Philadelphia: Temple University Press.

------. 1997. "A Rebuttal on Health." In What is Disease?, edited by James M. Humber and Rober F. Almeder, 3-134. Totowa, New Jersey: Humana Press.

------. 2012 (manuscript). “Clinical Normality.” Available at http://www.philosophie.unihamburg.de/Schramme/Konferenzen_/Boorse\%20Clinical\%20Normality.pdf . Accessed 2014-1-18.

Chobanian, Aram V., George L. Bakris, Henry R. Black, William C. Cushman, Lee A. Green, Joseph L. Izzo, Jr., Daniel W. Jones, Barry J. Materson, Suzanne Oparil, Jackson T. Wright, Jr., Edward J. Roccella, and the National High Blood Pressure Education Program Coordinating Committee. 2003. "The Seventh Report of the Joint National Committee on Prevention, Detection, Evaluation, and Treatment of High Blood Pressure: The JNC 7 Report." JAMA 289 (19):2560-72.

Clouser, K. Danner, Charles M. Culver, and Bernard Gert. 1981. "Malady: a New Treatment of Disease." Hastings Center Report 11 (3):29-37.

Daniels, Norman. 2008. Just Health: Meeting Health Needs Fairly. Cambridge: Cambridge University Press.

Croswell, Jennifer M., Otis W. Brawley, and Barnett S. Kramer. 2011. "Prevention and Early Detection of Cancer." In Harrison's Principles of Internal Medicine, 18th Ed., edited by D. Longo, D. Kasper, J. Jameson, A. Fauci, S. Hauser and J. Loscalzo, New York: McGraw-Hill Professional. 655-662.

Erbas, Bircan, Elena Provenzano, Jane Armes, and Dorota Gertig. 2006. "The natural History of Ductal Carcinoma in situ of the Breast: a Review." Breast Cancer Research and Treatment 97 (2):135-44..

Ernster, Virginia L., John Barclay, Karla Kerlikowske, Deborah Grady, and I. Craig Henderson. 1996. "Incidence of and Treatment for Ductal Carcinoma in situ of the Breast." JAMA 275 (12):913-8.

Eusebi, V., E. Feudale, M. Foschini, A. Micheli, A. Conti, C. Riva, S. Di Palma, and F. Rilke. 1994. "Long-term Follow-up of in situ Carcinoma of the Breast." Seminars in Diagnostic Pathology 11 (3):223-35.

Greene, Jeremy. 2007. Prescribing by the numbers: drugs and the definition of disease. Baltimore: Johns Hopkins Univ. Press.

Hausman, Daniel M. 2012. "Health, Naturalism, and Functional Efficiency." Philosophy of Science 79 (4):519-541.

Kuerer, Henry M., Constance T. Albarracin, Wei T. Yang, Robert D. Cardiff, Abenaa M. Brewster, W. Fraser Symmans, Nola M. Hylton, Lavinia P. Middleton, Savitri Krishnamurthy, George H. Perkins, Gildy Babiera, Mary E. Edgerton, Brian J. Czerniecki, Banu K. Arun and Gabriel N. Hortobagyi. 2009. "Ductal Carcinoma in situ: State of the Science and Roadmap to Advance the Field." Journal of Clinical Oncology 27 (2):279-88.

Miller, Franklin G., and Howard Brody. 2001. "The Internal Morality of Medicine: An Evolutionary Perspective." Journal of Medicine and Philosophy 26 (6):581-599. 
National Cholesterol Education Program (NCEP), Expert Panel on Detection, Evaluation, and Treatment of High Blood Cholesterol in Adults (Adult Treatment Panel III). 2002. "Third Report of the National Cholesterol Education Program (NCEP) Expert Panel on Detection, Evaluation, and Treatment of High Blood Cholesterol in Adults (Adult Treatment Panel III) Final Report." Circulation 106:3143-3421.

Omer, Zehra B., E. Shelley Hwang, Laura J. Esserman, Rebecca Howe, and Elissa M. Ozanne. 2013. "Impact of Ductal Carcinoma in situ Terminology on Patient Treatment Preferences." JAMA Internal Medicine 173 (19):1830-1.

Page, David L., William D. Dupont, Lowell W. Rogers, Marie Landenberger. 1982. "Intraductal Carcinoma of the Breast: Follow-up after Biopsy Only." Cancer 49 (4):751-8.

Schwartz, Peter H. 2007a. "Decision and Discovery in Defining 'Disease'." In Establishing Medical Reality: Essays in the Metaphysics and Epistemology of Biomedical Science, edited by H. Kincaid and J. McKitrick, 47-64. Dordrecht: Springer.

-------. 2007b. "Defining Dysfunction: Natural Selection, Design, and Drawing a Line." Philosophy of Science 74 (7):364-385.

------. 2008. "Risk and Disease." Perspectives in Biology and Medicine 51 (3):320-334.

U.S. Preventive Services Task Force. 2008. U.S. Preventive Services Task Force Procedure Manual. edited by Agency of Healthcare Research and Quality (AHRQ). AHRQ Publication No. 08-05118-EF. Available at: http://www.uspreventiveservicestaskforce.org/uspstf08/methods/procmanual.pdf. Accessed July 7, 2013.

Vickers, Andrew J., Ethan Basch, and Michael W. Kattan. 2008. "Against Diagnosis." Annals of Internal Medicine 149 (3): 200-3.

Wakefield, Jerome C. 1992a. "The Concept of Mental Disorder: On the Boundary between Biological Facts and Social Values." American Psychologist 47 (3):373388.

-------. 1992b. "Disorder as Harmful Dysfunction: A Conceptual Critique of DSM-III's Definition of Mental Disorder." Psychological Review 99 (2):232-247.

Welch, H. Gilbert, and William C. Black. 1997. "Using Autopsy Series to Estimate the Disease "Reservoir" for Ductal Carcinoma in situ of the Breast: How Much More Breast Cancer can we Find?" Annals of Internal Medicine 127 (11):1023-8.

Westin, Steinar, and Iona Heath. 2005. "Thresholds for Normal Blood Pressure and Serum Cholesterol." BMJ 330 (7506):1461-1462. 